\title{
Stabilizer for Hopf Algebra Actions
}

\author{
Min Yan Yongchang Zhu \\ Dept. of Math., HKUST, Clear Water Bay, Hong Kong
}

Abstract. The definition of stabilizer and orbit for Hopf algebra action is given, and a duality theorem on stabilizer is proved.

\section{Introduction.}

Let $H$ be a Hopf algebra. An $H$-module algebra is an associative algebra on which $H$ acts compatibly. Consider the case $H$ is a group algebra $\mathbb{C} G$. A $G$-set gives arise to a commutative $\mathbb{C} G$-module algebra by taking the algebra of the complex functions on $S$. A non-commutative $\mathbb{C} G$-module algebra may be viewed as the function algebra on a hypothetical $G$-space. For a general Hopf algebra $H$, it is instructive to view the notion of $H$-module algebra as a generalization of $G$-set, generalizing both group $G$ and set $S$ to the quantum case.

The purpose of this work is to show that the concept of stabilizer for group action also exists for Hopf algebra action. Unlike the case of group actions, a stabilizer of an $\mathrm{H}$-module algebra is in general not a Hopf subalgebra, but it is a module algebra for the dual Hopf algebra $H^{*}$. More specifically, for a left $H$-module algebra $M$ together with an $M$-module $V$, the stabilizer of the pair $(M, V)$ is another pair $\left(M^{\prime}, V^{\prime}\right)$, where $M^{\prime}$ is a right $H^{*}$-module algebra and $V^{\prime}$ is an $M^{\prime}$-module. Similarly the stabilizer is defined for a pair $\left(M^{\prime}, V^{\prime}\right)$ for $H^{*}$. Our main theorem asserts that the stabilizer of the stabilizer of $(M, V)$ is actually isomorphic to $(M, V)$ itself under certain assumptions on $(M, V)$. This theorem generalizes the known fact that the structure of a transitive $G$-set $S$ is determined by the stabilizer of a point $p \in S$. It also implies that if $H$ is semisimple then the set of isomorphism classes of pairs $(M, V)$ for $H$ satisfying certain properties is $1-1$ correspondent to the set of isomorphism classes of pairs $(M, V)$ for $H^{*}$ satisfying the similar properties.

It might be interesting to compare our result on Hopf algebra actions with some result in [D1] on Poisson homogeneous spaces. Let us first recall certain analogy between Poisson Lie groups and Hopf algebras. If $A_{t}$ is a one parameter family of associative algebras with $A_{0}$ being the commutative algebra of smooth 
functions on $M$, then the first order derivative of $A_{t}$ at $t=0$ gives more or less $M$ a Poisson manifold structure. The resulting Poisson manifold $M$ is known as the classical limit of $A_{t}$. If $A_{t}$ are Hopf algebras, its classical limit is a Poisson Lie group. It often happens that some constructions for Poisson Lie groups have analogs for Hopf algebras, though not every Hopf algebra is a deformation of a group. The double of a Poisson Lie group and the Drinfeld double of a Hopf algebra is such an example [D2]. The results in [D1] imply that for a simply connected Poisson Lie group $G$, the set of isomorphism classes of pairs $(M, S)$, where $M$ is a Poisson homogeneous space of $G$ and $S$ is a symplectic leaf on $M$, is $1-1$ correspondent to the set of isomorphism classes of pairs $\left(M^{\prime}, S^{\prime}\right)$ for the simply connected dual Poisson Lie group $G^{*}$. A pair $(M, S)$ for $G$ and its correspondent pair $\left(M^{\prime}, S^{\prime}\right)$ for $G^{*}$ may be defined as stabilizers of each other. Our result is clearly the analog of this part of the result in [D1].

From algebraic perspective, our result can be described as follows. Suppose $R$ is a simple algebra and $H$ is a finite dimensional Hopf algebra over a field. Then there is a 1-1 correspondence between semisimple right $H^{*}$-comodule subalgebras in $R \otimes H^{*}$ and semisimple left $H$-module subalgebras in $H \otimes R$. However, we feel the analogy with Poisson homogeneous spaces might be more interesting.

In Section 2 we shall recall the basic definitions related to the module algebras of a Hopf algebra. In section 3 we define (construct) the orbit module algebra for Hopf algebra action. In section 4 we define (construct) the stabilizer for a pair $(M, V)$ as above. In section 5 we prove our duality theorem for stabilizers. We shall assume all the algebras considered in the paper are finite dimensional.

\section{Module algebras.}

Let $H$ denote a finite dimensional Hopf algebra over a given field $k$ with comultiplication $\Delta$, antipode $S$ and counit $\epsilon$. Let $H^{*}$ denote the dual Hopf algebra of $H$. We will write the pairing of $H$ and $H^{*}$ as $\langle a, f\rangle$ for $a \in H$ and $f \in H^{*}$.

A (left or right) $H$-module algebra is an associative algebra with compatible $H$-module structure. A subspace $I \subset M$ is a module ideal if $I$ is an $H$-submodule and a two-sided ideal of the algebra $M$. In this case, the quotient space $M / I$ is also an $H$-module algebra. The dual notion of $H$-comodule algebras may 
be similarly defined, and for finite dimensional modules, the duality operation provides various equivalences between modules and comodules over $H$ and $H^{*}$.

We note that $H^{*}$ is a left $H$-module-algebra under the action $\rightarrow$ given by

$$
a \rightarrow f=\left\langle a, f_{(2)}\right\rangle f_{(1)}, \quad \text { or } \quad\langle b, a \rightarrow f\rangle=\langle b a, f\rangle .
$$

$H^{*}$ is also a right $H$-module-algebra under the action $\angle$ given by

$$
f<a=\left\langle a, f_{(1)}\right\rangle f_{(2)}, \quad \text { or } \quad\langle b, f<a\rangle=\langle a b, f\rangle .
$$

The two actions $\rightarrow$ and $<$ clearly commute with each other. Moreover, since $H$ is assumed finite dimensional, the two actions, considered as subalgebras of $\operatorname{End}\left(H^{*}\right)$, are centralizers to each other. Simialrly we have actions $\rightarrow$ and $\leftarrow$ of $H^{*}$ on $H$. They are also centralizers to each other.

Hopf algebras and (co)module algebras over it are analogues of groups and sets acted by groups. For a finite group $G$, the group algebra $k G$ is a finite dimensional Hopf algebra. A left $k G$-module algebra is an algebra with a left action of $G$ as symmetry. If $S$ is a $G$-set, the algebra $F(S)$ of $k$-valued functions on $S$ is a commutative $k G$-module algebra. For $P \in S$, let $I_{P}$ be the function that takes value 1 at $P$ and 0 elsewhere, then $\left\{I_{P} \mid P \in S\right\}$ is a basis of $F(S)$. The multiplication is $I_{P_{1}} I_{P_{2}}=\delta_{P_{1}, P_{2}} I_{P_{1}}$. The action of $k G$ on $F(S)$ is $g \cdot I_{P}=I_{g P}$.

The dual Hopf algebra of $k G$, denoted by $(k G)^{*}$, has a basis $\left\{I_{g} \mid g \in G\right\}$ with multiplication $I_{g} I_{h}=\delta_{g, h} I_{g}$ and comultiplication $\Delta I_{g}=\sum_{x \in G} I_{x} \otimes I_{x^{-1} g}$. A left $(k G)^{*}$-module algebra is precisely a $G$-graded algebra. Recall a $G$-graded algebra is $M=\oplus_{g \in G} M_{g}$ such that $1 \in M_{e}$ and $M_{g} M_{h} \subset M_{g h}$ (some of $M_{g}$ may be $\{0\})$. The action of $(k G)^{*}$ on $M=\oplus_{g \in G} M_{g}$ is given by $I_{g} v=v$ if $v \in M_{g}$ and $I_{g} v=0$ for $v \in M_{h}, h \neq g$. A $G$-graded algebra can be also viewed as right $(k G)^{*}$-module algebra by the right action $v I_{g}=v$ for $v \in M_{g^{-1}}$ and $v I_{g}=0$ for $v \in M_{h}, h \neq g^{-1}$. If $G_{1} \subset G$ is a subgroup, the group algebra $M=k G_{1}$ is a $G$-graded algebra by setting $M_{g}=k g$ for $g \in G_{1}$ and $M_{g}=0$ for $g \notin G_{1}$. Therefore $k G_{1}$ is both a left and a right $(k G)^{*}$-module algebra.

Our constructions and theorems will be tested against group actions on sets.

\section{Section 3. Orbits.}


In this section, we define for a (left) $H$-module algebra $M$ together with a representation $M \rightarrow \operatorname{End}(V)$, a new $H$-module algebra called the orbit of $V$. And we show that our definition generalizes the orbit for group action.

Let us first make a few observations. If $M$ is a module algebra of $H, A$ is an arbitrary algebra, then the tensor product algebra $A \otimes M$ is also an $H$-module algebra by the action $a \cdot(b \otimes m)=b \otimes(a \cdot m)$ for $a \in H, b \otimes m \in A \otimes M$. In particular, since $H^{*}$ is an $H$-module algebra under the action $\rightarrow, A \otimes H^{*}$ is an $H$-module algebra for any algebra $A$. The next proposition says that any $H$-module algebra is an $H$-module subalgebra of a standard one $A \otimes H^{*}$.

Proposition 3.1. Suppose that $M$ is an $H$-module algebra. Then the corresponding right $H^{*}$-comodule structure map $\delta: M \rightarrow M \otimes H^{*}$ is an $H$-module algebra embedding.

In the proposition, the $H$-module algebra structure on $M \otimes H^{*}$ is given by the algebra structure on $M$ and the left $H$-module algebra structure $\rightarrow$ on $H^{*}$. In particular, if $M \rightarrow A$ is an algebra morphism, then $M \otimes H^{*} \rightarrow A \otimes H^{*}$ is an $H$-module algebra morphism.

$\delta$ is in fact split injective because it is the structure map for the $H^{*}$-comodule $M$. It is straightforward to check that $\delta$ is a morphism of $H$-module algebras.

Let $V$ be an $M$-module. Let $\pi: M \rightarrow \operatorname{End}(V)$ be the corresponding algebra morphism. Then the composition

$$
\Pi_{V}: M \stackrel{\delta}{\longrightarrow} M \otimes H^{*} \stackrel{\pi \otimes \mathrm{id}}{\longrightarrow} \operatorname{End}(V) \otimes H^{*}
$$

is an $H$-module algebra morphism. The kernel $\operatorname{Ker}\left(\Pi_{V}\right)$ is a module ideal of $M$. We call the quotient $H$-module algebra $M / \operatorname{Ker}\left(\Pi_{V}\right)$ the orbit module algebra of $V$.

Let us check that our definition of orbit agrees with the orbit of a group action on a set.

Let $G$ be a finite group, $S$ be a finite $G$-set. The algebra $F(S)$ of functions on $S$ is a $\mathbb{C} G$-module algebra (see $\S 2$ ). The corresponding comodule map $\delta$ : $F(S) \rightarrow F(S) \otimes(\mathbb{C} G)^{*}$ is

$$
\sum_{P \in S} k_{P} I_{P} \mapsto \sum_{P \in S} \sum_{g \in G} k_{P} I_{g P} \otimes I_{g}
$$


A given point $Q \in S$ defines a one dimensional $F(S)$-module by

$$
\pi_{Q}: F(S) \rightarrow \mathbb{C}, \quad \sum_{P \in S} k_{P} I_{P} \mapsto k_{Q}
$$

It is easy to see that the kernel of the composition $\Pi=\left(\pi_{Q} \otimes \mathrm{id}\right) \delta: F(S) \rightarrow$ $F(S) \otimes(\mathbb{C} G)^{*} \rightarrow \mathbb{C} \otimes(\mathbb{C} G)^{*}=(\mathbb{C} G)^{*}$ is

$$
\operatorname{Ker}(\Pi)=\left\{\sum_{P \in S} k_{P} I_{P} \mid k_{P}=0 \text { if } P \text { is in the orbit of } Q\right\} .
$$

So the orbit module algebra for $\pi_{Q}, F(S) / \operatorname{Ker}(\Pi)$, is isomorphic to the $F\left(O_{Q}\right)$ (where $O_{Q}$ is the orbit of $Q$ ).

Similarly, for $T=\left\{Q_{1}, \ldots, Q_{n}\right\} \subset S, F(S)$ has an $n$-dimensional module $\mathbb{C}^{n}$ given by

$$
\pi_{T}: F(S) \rightarrow \operatorname{End}\left(\mathbb{C}^{n}\right)=M_{n \times n}(\mathbb{C}), \quad \sum_{P \in S} k_{P} I_{P} \mapsto \operatorname{diag}\left(k_{Q_{1}}, k_{Q_{2}}, \ldots, k_{Q_{n}}\right) .
$$

It is not hard to prove that the orbit module algebra for $\pi_{T}$ is $F\left(O_{T}\right)$, where $O_{T}=O_{Q_{1}} \cup O_{Q_{2}} \cup \cdots \cup O_{Q_{n}}$ is the orbit of $T$.

\section{Section 4. Stabilizer.}

In this section we consider a pair $(M, V)$, where $M$ is a left $H$-module algebra, and $V$ is an $M$-module. We will construct another pair $\left(M^{\prime}, V^{\prime}\right)$, where $M^{\prime}$ is a right $H^{*}$-module algebra, and the same space $V$ has an $M^{\prime}$-module structure called $V^{\prime}$. The pair $\left(M^{\prime}, V^{\prime}\right)$ is defined to be the stabilizer of $(M, V)$.

Since our construction uses the smash product, we brielfly recall its definition. Let $M$ be a left $H$-module algebra. Then the smash product algebra (see e.g. [M]) $M \# H$ is $M \otimes H$ with product

$$
(m \otimes a)(n \otimes b)=\sum m\left(a_{(1)} \cdot n\right) \otimes a_{(2)} b .
$$

Moreover, $M \# H$ is a left $H^{*}$-module algebra by

$$
f \cdot(m \otimes a)=m \otimes(f \rightarrow a) .
$$

Similarly, if $M$ is a right $H$-module algebra, then the smash product $H \# M$ is $H \otimes M$ with product

$$
(a \otimes m)(b \otimes n)=\sum a b_{(1)} \otimes\left(m \cdot b_{(2)}\right) n .
$$


$H \# M$ is a right $H^{*}$-module algebra by

$$
(a \otimes m) \cdot f=(a<f) \otimes m .
$$

The action $\rightarrow$ makes $H$ into a left $H^{*}$-module algebra. The resulting smash product $\mathcal{H}(H)=H \# H^{*}$ is called the Heisenberg double of $H$. Alternatively, $\left\llcorner\right.$ makes $H^{*}$ into a right $H$-module algebra, which gives rise to another smash product denoted also by $\mathcal{H}(H)$. Fortunately there is no confusion here because the products are the same:

$$
(a \otimes g)(b \otimes h)=\sum\left\langle b_{(2)}, g_{(1)}\right\rangle a b_{(1)} \otimes g_{(2)} h
$$

From the above discussion we know that $\mathcal{H}(H)$ is a left $H$-module algebra and a right $H^{*}$-module algebra. Moreover, it is easy to see that the natural inclusions

$$
H \subset \mathcal{H}(H), \quad a \mapsto a \otimes 1
$$

are respectively left $H$ and right $H^{*}$ module algebra embeddings.

Now we are ready to construct the stabilizer of a pair $(M, V)$. Consider the $H$-module algebra morphism

$$
\Pi_{V}: M \stackrel{\delta}{\longrightarrow} M \otimes H^{*} \stackrel{\pi \otimes 1}{\longrightarrow} \operatorname{End}(V) \otimes H^{*} \stackrel{i n c l}{\longrightarrow} \operatorname{End}(V) \otimes \mathcal{H}(H)
$$

which is the morphism (3.1) extended by the inclusion (4.2) and is still denoted as $\Pi_{V}$. Then we construct

$$
M^{\prime}=\left\{x \in \operatorname{End}(V) \otimes H \mid x \quad \text { commutes with all } y \in \Pi_{V}(M)\right\} .
$$

Clearly $M^{\prime}$ is an associative subalgebra of $\operatorname{End}(V) \otimes H$. Moreover, since the action $\rightarrow$ of $H^{*}$ on $\operatorname{End}(V) \otimes \mathcal{H}(H)$ has the trivial restriction on the subspace $\operatorname{End}(V) \otimes H^{*}$ and subsequently on $\Pi_{V}(M), M^{\prime}$ is also a right $H^{*}$-submodule of $\operatorname{End}(V) \otimes H$. So we have the following proposition. 
Proposition 4.1. $M^{\prime}$ defined as above is a right $H^{*}$-module algebra.

The space $V$ has an $M^{\prime}$-module structure as follows. The counit $\epsilon: H \rightarrow k$ induces an algebra homomorphism $1 \otimes \epsilon: \operatorname{End}(V) \otimes H \rightarrow \operatorname{End}(V)$. This restricts to an algebra homomorphism $M^{\prime} \rightarrow \operatorname{End}(V)$. We denote this $M^{\prime}$-module by $V^{\prime}$.

We call the pair $\left(M^{\prime}, V^{\prime}\right)$ the stabilizer of $(M, V)$.

Similarly, if we start with a pair $(N, V)$, where $N$ is a right $H^{*}$-module algebra $N$ and $V$ is an $N$-module, we can construct a pair $\left(N^{\prime}, V^{\prime}\right)$, where $N^{\prime}$ is a left $H$-module algebra and $V^{\prime}$ is a $N^{\prime}$-module. Specifically, let $\delta: N \rightarrow H \otimes N$ be the left $H$-comodule structure associated to the right $H^{*}$-module structure. Let $\tau: H \otimes N \rightarrow N \otimes H$ be the switching map. Let $\pi: N \rightarrow \operatorname{End}(V)$ be the structure map of $N$-module $V$. Consider the composition

$$
\Pi_{V}: N \stackrel{\delta}{\rightarrow} H \otimes N \stackrel{\tau}{\rightarrow} N \otimes H \stackrel{\pi \otimes 1}{\longrightarrow} \operatorname{End}(V) \otimes H \stackrel{i n c l}{\longrightarrow} \operatorname{End}(V) \otimes \mathcal{H}(H)
$$

and construct

$$
N^{\prime}=\left\{x \in \operatorname{End}(V) \otimes H^{*} \mid x \text { commutes with all } y \in \Pi_{V}(N)\right\} .
$$

Then $N^{\prime}$ is a left $H$-module algebra and the algebra morphism $1 \otimes \epsilon: \operatorname{End}(V) \otimes$ $H^{*} \rightarrow \operatorname{End}(V)$ restricts to $N^{\prime}$ and gives an $N^{\prime}$-module structure on $V$, we denote this $N^{\prime}$-module by $V^{\prime}$. We call $\left(N^{\prime}, V^{\prime}\right)$ the stabilizer of $(N, V)$.

We remark that since there is a natural correspondence between left module algebras for $H\left(H^{*}\right)$ and right module algebras for $H^{*}(H)$ by use of $S$, we can modify our definition so that only the notion of left module algebra appears.

Consider the case that a finite group $G$ acts on a finite set $S, F(S)$ is a left module algebra of $\mathbb{C} G$. For $Q \in S$, let $\pi: F(S) \rightarrow V=\mathbb{C}$ be the associated 1-dimensional module given by $\pi_{Q}: \sum_{P \in S} k_{P} I_{P} \mapsto k_{Q}$. The map $\Pi: F(S) \rightarrow$ $\operatorname{End}(V) \otimes \mathcal{H}(H)=\mathcal{H}(H)$ is given explicitely as

$$
\Pi_{V}: I_{P} \mapsto \sum_{g \in G, g P=Q} I_{g} .
$$

$M^{\prime}$ is by definition the subspace of $\operatorname{End}(V) \otimes H=H$ that consists of elements commuting with all elements of form $\sum_{g \in G, g P=Q} I_{g}$, i.e., $\sum_{h \in G} k_{h} h \in M^{\prime}$ iff

$$
\left(\sum_{h \in G} k_{h} h \otimes 1\right)\left(1 \otimes \sum_{g \in G, g P=Q} I_{g}\right)=\left(1 \otimes \sum_{g \in G, g P=Q} I_{g}\right)\left(\sum_{h \in G} k_{h} h \otimes 1\right),
$$


for every $P \in S$, where (4.5) is an identity in $\mathcal{H}(H)$. Using (4.5) it is easy to verify that $M^{\prime}$ is $\mathbb{C} G_{Q}$, where $G_{Q}$ is the stabilizer of $Q$. The $V^{\prime}$ is just the one dimensional module of $\mathbb{C} G_{Q}$ given by the counit $\epsilon$. So the stabilizer of $\left(F(S), \pi_{Q}\right)$ is $\left(\mathbb{C} G_{Q}, \epsilon\right)$ (recall that $G_{Q}$ is a right $(\mathbb{C} G)^{*}$-module algebra by $\S 2$ ).

\section{Section 5. Duality on Stabilizers.}

We continue to assume that $H$ is a finite dimensional Hopf algebra, $M$ is a left $H$-module algebra and $V$ is an $M$-module. We prove in this section that $\left(M^{\prime \prime}, V^{\prime \prime}\right)$, the stabilizer of the stabilizer of $(M, V)$, is isomorphic to $(M, V)$ under certain conditions.

Recall that in the definitions of orbit and stabilizer, we used the map $\Pi_{V}$ : $M \rightarrow \operatorname{End}(V) \otimes \mathcal{H}(H)$. We call $(M, V)$ transitive if $\Pi_{V}$ is injective.

A key step in our proof is to show that $M^{\prime \prime}$ is isomorphic to the double centralizer of $\Pi_{V}(M)$ in $\operatorname{End}(V) \otimes \mathcal{H}(H)$. For this purpose we need to understand the relationship between $M^{\prime}$ and the centralizer

$$
C(M, V)=\left\{x \in \operatorname{End}(V) \otimes \mathcal{H}(H) \mid x \text { commutes with all } y \in \Pi_{V}(M)\right\}
$$

of $\Pi_{V}(M)$ in $\operatorname{End}(V) \otimes \mathcal{H}(H)$.

We identify $\operatorname{End}(V) \otimes \mathcal{H}(H)$ with $\operatorname{End}(V) \otimes \operatorname{End}(H)$ by the isomorphism of algebras

$$
\lambda: \mathcal{H}(H) \rightarrow \operatorname{End}(H), \quad \lambda(a \otimes f)(x)=a(f \rightarrow x)
$$

Proposition 5.1. Let $R: H^{*} \rightarrow \operatorname{End}(H)$ be the anti-algebra embedding given by

$$
R(f) x=x<f
$$

Using the identification (5.1) and the inclusion $1 \otimes i d$, we view $R\left(H^{*}\right)$ as a subalgebra of End $\otimes \mathcal{H}(H)$. Then $C(M, V)=R\left(H^{*}\right) M^{\prime}$.

We give a corollary of this proposition before proving it.

Corollary 5.1. The algebra $M^{\prime \prime}$ is the same as the double centralizer of $\Pi_{V}(M)$ in $\operatorname{End}(V) \otimes \mathcal{H}(H)$.

Proof. By the definition in $\S 4$,

$$
M^{\prime \prime}=\left\{x \in \operatorname{End}(V) \otimes H^{*} \mid x \text { commutes with all } y \in \Pi_{V^{\prime}}\left(M^{\prime}\right)\right\} .
$$


Using the construction of $\left(M^{\prime}, V^{\prime}\right)$, we can prove that $\Pi_{V^{\prime}}\left(M^{\prime}\right)$ is the same as $M^{\prime} \subset \operatorname{End}(V) \otimes \mathcal{H}(H)$ (essentially, this boils down to $(\epsilon \otimes 1) \delta=i d$ in $H$ ). So we have

$$
M^{\prime \prime}=\left\{x \in \operatorname{End}(V) \otimes H^{*} \subset \operatorname{End}(V) \otimes \mathcal{H}(H) \mid x \text { commutes with } M^{\prime}\right\} .
$$

By Proposition 5.1, the double centralizer of $M$ in $\operatorname{End}(V) \otimes \mathcal{H}(H)$ is the intersection of the centralizer of $M^{\prime}$ in $\operatorname{End}(V) \otimes \mathcal{H}(H)$ and the centralizer of $R\left(H^{*}\right)$ in $\operatorname{End}(V) \otimes \mathcal{H}(H)$. Because under the identification (5.1), $R\left(H^{*}\right)$ consists of operators " $\angle f$ ", $H^{*} \subset \mathcal{H}(H)$ consists of operators " $f \longrightarrow$ ", so the centralizer of $R\left(H^{*}\right)$ in $\mathcal{H}(H)$ is $H^{*}$, and the centralizer of $R\left(H^{*}\right)$ in $\operatorname{End}(V) \otimes \mathcal{H}(H)$ is $\operatorname{End}(V) \otimes H^{*}$. Therefore $M^{\prime \prime}$ is the same as the double centralizer of $M$ in $\operatorname{End}(V) \otimes \mathcal{H}(H)$.

Our proof of Proposition 5.1 makes use of the theory of Hopf modules which we briefly recall as follows (see e.g. [M] for detail). A right $H^{*}$-Hopf module is a vector space such that

(1) $V$ is a left $H$-module;

(2) $V$ is a right $H^{*}$-module;

(3) The map $V \rightarrow V \otimes H^{*}$ given by the left $H$-module structure is a right $H^{*}$-module morphism.

The third condition means that

$$
a \cdot(v \cdot f)=\sum\left(a_{(1)} \cdot v\right) \cdot\left(a_{(2)} \rightarrow f\right) .
$$

The next Lemma introduces a right $H^{*}$-Hopf module structure on $\operatorname{End}(H)$. This can be translated to a right $H^{*}$-Hopf module structure on $\mathcal{H}(H)$ by $(5.1)$.

Lemma 5.1. Let $R: H \rightarrow \operatorname{End}(H)$ be the antimorphim given by $R(a) x=x a$, and $R: H^{*} \rightarrow \operatorname{End}(H)$ be the antimorphism given by $R(f) x=x<f$. $\operatorname{End}(H)$ is a right $H^{*}$-Hopf module with the left $H$-action

$$
a \bullet T=\sum R\left(S a_{(2)}\right) T R\left(a_{(1)}\right), \quad a \in H, \quad T \in \operatorname{End}(H),
$$

and right $H^{*}$-action

$$
T \bullet f=R(f) T, \quad f \in H^{*}, \quad T \in \operatorname{End}(H) .
$$


Proof: Consider the linear isomorphism

$$
F: H \otimes H^{*} \rightarrow \operatorname{End}(H), \quad F(a \otimes f)(x)=(a x)<f
$$

(note that $F$ is not an algebra morphism). It can be verified that

$$
a \bullet F(b \otimes g)=F(b \otimes(a \rightarrow g)), \quad F(b \otimes g) \bullet f=F(b \otimes g f) .
$$

We see that under the identification $F$, our actions become a left $H$-action and a right $H^{*}$-action on the $H^{*}$ factor in $H \otimes H^{*}$. This is a right $H^{*}$-Hopf module structure (see e.g. $[\mathrm{M}]$ ). This proves the lemma.

We denote the antimorphism

$$
H \stackrel{R}{\longrightarrow} \operatorname{End}(H) \stackrel{\lambda^{-1}}{\longrightarrow} \mathcal{H}(H) \stackrel{1 \otimes i d}{\longrightarrow} \operatorname{End}(V) \otimes \mathcal{H}(H)
$$

and antimorphism

$$
H^{*} \stackrel{R}{\longrightarrow} \operatorname{End}(H) \stackrel{\lambda^{-1}}{\longrightarrow} \mathcal{H}(H) \stackrel{1 \otimes i d}{\longrightarrow} \operatorname{End}(V) \otimes \mathcal{H}(H)
$$

by $\bar{R}$. Then by Lemma 5.1 , the following is a right $H^{*}$-Hopf module structure on $\operatorname{End}(V) \otimes \mathcal{H}(H)$ :

$$
a \bullet x=\sum \bar{R}\left(S a_{(2)}\right) x \bar{R}\left(a_{(1)}\right), \quad x \bullet f=\bar{R}(f) x .
$$

Lemma 5.2. $C(M, V) \subset \operatorname{End}(V) \otimes \mathcal{H}(H)$ is invariant under the actions (5.4), so that $C(M, V)$ is a right $H^{*}$-Hopf module.

Proof: We first prove the following formula

$$
R(a) \lambda(1 \otimes f)=\sum \lambda\left(1 \otimes\left(S a_{(2)} \rightarrow f\right)\right) R\left(a_{(1)}\right)
$$

in $\operatorname{End}(H)$. In fact, applying both sides to $b \in H$ gives us

$$
\begin{aligned}
\sum\left(\lambda\left(1 \otimes\left(S a_{(2)} \rightarrow f\right)\right) R\left(a_{(1)}\right)\right)(b) & =\sum\left(S a_{(2)} \rightarrow f\right) \rightarrow b a_{(1)} \\
& =\sum\left\langle b_{(2)} a_{(2)}, S a_{(3)} \rightarrow f\right\rangle b_{(1)} a_{(1)} \\
& =\sum\left\langle b_{(2)} a_{(2)} S a_{(3)}, f\right\rangle b_{(1)} a_{(1)} \\
& =\sum\left\langle b_{(2)}, f\right\rangle b_{(1)} a \\
& =(f \rightarrow b) a \\
& =(R(a) \lambda(1 \otimes f))(b) .
\end{aligned}
$$


Translated into an equality in $\operatorname{End}(V) \otimes \mathcal{H}(H),(5.5)$ becomes

$$
\bar{R}(a)(u \otimes 1 \otimes f)=\sum\left(u \otimes 1 \otimes\left(S a_{(2)} \rightarrow f\right)\right) \bar{R}\left(a_{(1)}\right)
$$

Now for $y=\sum u_{i} \otimes 1 \otimes f_{i} \in \Pi_{V}(M), x \in C(M, V)$ and $a \in H$,

$$
\begin{aligned}
(a \bullet x) y & =\sum \bar{R}\left(S a_{(2)}\right) x \bar{R}\left(a_{(1)}\right)\left(u_{i} \otimes 1 \otimes f_{i}\right) \\
& \stackrel{(5.6)}{=} \sum \bar{R}\left(S a_{(3)}\right) x\left(u_{i} \otimes 1 \otimes\left(S a_{(2)} \rightarrow f_{i}\right)\right) \bar{R}\left(a_{(1)}\right) \\
& \stackrel{?}{=} \sum \bar{R}\left(S a_{(3)}\right)\left(u_{i} \otimes 1 \otimes\left(S a_{(2)} \rightarrow f_{i}\right)\right) x \bar{R}\left(a_{(1)}\right) \\
& \stackrel{(5.6)}{=} \sum\left(u_{i} \otimes 1 \otimes\left(S^{2} a_{(3)} \rightarrow S a_{(2)} \rightarrow f_{i}\right)\right) \bar{R}\left(S a_{(4)}\right) x \bar{R}\left(a_{(1)}\right) \\
& =\sum\left(u_{i} \otimes 1 \otimes\left(S^{2} a_{(3)} S a_{(2)} \rightarrow f_{i}\right)\right) \bar{R}\left(S a_{(4)}\right) x \bar{R}\left(a_{(1)}\right) \\
& =\sum\left(u_{i} \otimes 1 \otimes f_{i}\right) \bar{R}\left(S a_{(2)}\right) x \bar{R}\left(a_{(1)}\right) \\
& =y(a \bullet x),
\end{aligned}
$$

where ? follows from the fact that $\sum u_{i} \otimes 1 \otimes\left(b \rightarrow f_{i}\right) \in \Pi_{V}(M)$, since $\Pi_{V}(M)$ is closed under the $H$-action $\rightarrow$. This proves the invariance of $C(M, V)$ under the $H$-action.

For the invariance under the right $H^{*}$-action, we need the following formula

$$
R(g) \lambda(1 \otimes f)=\lambda(1 \otimes f) R(g)
$$

In fact, applying both sides to $b \in H$ gives us $f \rightarrow b<g$. Translated into $\operatorname{End}(V) \otimes \mathcal{H}(H)$, we obtain

$$
\bar{R}(g)(1 \otimes f)=(1 \otimes f) \bar{R}(g)
$$

For $x, y$ as above, $(x \bullet g) y=R(g) x y=R(g) y x=y R(g) x=y(x \bullet g)$.

Proof of Proposition 5.1: Let $C(M, V)^{H}$ be the $H$-invariant part of $C(M, V)$. The fundamental theorem of Hopf modules tells us that the right action map $C(M, V)^{H} \otimes H^{*} \rightarrow C(M, V)$ is a linear isomorphism (c.f. [M]). So we only need to show that

$$
M^{\prime}=C(M, V)^{H}
$$


i.e., $M^{\prime}$ is the invariants of $C(M, V)$ under the left $H$-action (5.4). Since $M^{\prime}=$ $C(M, V) \cap \operatorname{End}(V) \otimes H \otimes 1$, it suffices to prove that

$$
(\operatorname{End}(V) \otimes \mathcal{H}(H))^{H}=\operatorname{End}(V) \otimes H \otimes 1 .
$$

We first prove

$$
\operatorname{End}(V) \otimes H \otimes 1 \subset(\operatorname{End}(V) \otimes \mathcal{H}(H))^{H}
$$

By identifying $\operatorname{End}(V) \otimes \mathcal{H}(H)$ with $\operatorname{End}(V) \otimes \operatorname{End}(H)$ as before, $\operatorname{End}(V) \otimes H \otimes 1$ is identified with the subspace spanned by $T \otimes L(a)$ for $T \in \operatorname{End}(V)$ and $L(a) \in$ $\operatorname{End}(H)$, the left multiplication by $a \in H$. (5.9) then follows from the fact that $R(a)$ and $L(b)$ commutes.

By the fundamental theorem of Hopf modules, we know

$$
\operatorname{dim}(\operatorname{End}(V) \otimes \mathcal{H}(H))^{H}=\operatorname{dim}(\operatorname{End}(V) \otimes H) .
$$

Therefore (5.9) already implies (5.8).

Theorem 5.1. Let $M$ be a left $H$-module algebra and $V$ be an $M$-module. If $(M, V)$ is transitive and $M$ is semisimple, then the double stabilizer $\left(M^{\prime \prime}, V^{\prime \prime}\right)$ of $(M, V)$ is isomorphic to $(M, V)$.

Proof. By Corollary 5.1, $M^{\prime \prime}$ is the same as the double centralizer of $\Pi_{V}(M)$ in $\operatorname{End}(V) \otimes \mathcal{H}(H)$. By transitivity, $\Pi_{V}(M) \cong M$ is semisimple and, by algebra homomorphism (5.1), $\operatorname{End}(V) \otimes \mathcal{H}(H)$ is simple. The equality $\Pi_{V} \cong \Pi_{V}^{\prime \prime}$ (and consequently $M \cong M^{\prime \prime}$ ) then follows from the fact that the double centralizer of semisimple subalgebra of simple algebra is the subalgebra itself. It is clear that the isomorphism preserves the $H$-module algebra structure and carries $V$ to $V^{\prime \prime}$.

We believe Theorem 5.1 remains valid if the semisimplicity condition is replaced some other conditions, for example $M^{H}=k 1_{M}$.

We have the similar theorem for right $H^{*}$-module algebras.

Theorem 5.2. Let $M$ be a right $H^{*}$-module algebra and $V$ be an $M$-module. If $(M, V)$ is transitive and $M$ is semisimple, then the double stabilizer $\left(M^{\prime \prime}, V^{\prime \prime}\right)$ of $(M, V)$ is isomorphic to $(M, V)$. 
Theorem 5.3. If $H$ is semisimple, the stabilizer gives an one-to-one correspondence between the set of isomorphism classes of pairs $(M, V)$ where $M$ is a left $H$-module algebra and $V$ is an $M$-module such that $(M, V)$ is transitive and $M$ is semisimple and the set of isomorphism classes of pairs $\left(M^{\prime}, V^{\prime}\right)$ where $M^{\prime}$ is a right $H^{*}$-module algebra and $V^{\prime}$ is an $M^{\prime}$-module such that $\left(M^{\prime}, V^{\prime}\right)$ is transitive and $M^{\prime}$ is semisimple.

Proof. By Theorem 5.1 and Theorem 5.2, it suffices to prove that if $(M, V)$ is a pair for $H$ or $H^{*},(M, V)$ is transitive and $M$ is semisimple, then its stabilizer $\left(M^{\prime}, V^{\prime}\right)$ is transitive and $M^{\prime}$ is semisimple. We prove this for $H$. The proof for $H^{*}$ is similar. It is clear that $\left(M^{\prime}, V^{\prime}\right)$ is transitive. To prove $M^{\prime}$ is semisimple, we use $M^{\prime}=C(M, V)^{H}$ (see (5.7)). Since $M$ is semisimple, $C(M, V)$ is semisimple. $C(M, V)$ is an $H^{c o p}$-module algebra under the action (5.4)(where $H^{c o p}$ is $H$ with the opposite comultiplication). It follows from the lemma below that $M^{\prime}=$ $C(M, V)^{H}$ is semisimple.

Lemma 5.2. If $H$ is semisimple, $M$ is an $H$-module algebra and $M$ is semisimple, then $M^{H}$ is semisimple.

Proof. We prove that if $I \subset M^{H}$ is a left ideal, then there exists a complement left ideal $J \subset M^{H}$, i.e., $I \oplus J=M^{H}$. Recall that $M$ is an $M \# H$-module under the action $(m \otimes a) \cdot n=m(a \cdot n)$ for $m \otimes a \in M \# H$ and $n \in M$. It is clear that $M I \subset M$ is an $M \# H$-submodule. Since $M \# A$ is semisimple (see e.g. $[\mathrm{CF}]$ ), there exists another $M \# A$-submodule $N$ such that $M I \oplus N=M$. Therefore $(M I)^{H} \oplus N^{H}=M^{H}$. Using a left integral $\lambda \in H$ and the fact that $\epsilon(\lambda) \neq 0$, it can be prove that $(M I)^{H}=I$. And it is clear that $N^{H}$ is a left ideal of $M^{H}$.

\section{REFERENCES}

[CF] M.Cohen and D. Fischman, Hopf algebra actions, J. Algebra 100 (1986), 463-479.

[D1 ] V.G.Drinfeld, On Poisson Homogeneous Spaces of Poisson-Lie groups, Theo. Math. Phys. 95(2) (1993), 226-227.

[D2 ] V.G.Drinfeld, Quantum Groups, Proc. Int. Cong. Math, Berkeley (1986), 789-820.

[M] S. Montgomery, Hopf Algebras and Their Actions on Rings, AMS, 1993. 